\title{
High Rates of Tuberculosis in Patients Accessing HAART in Rural South Africa
}

\author{
Kogieleum Naidoo, MBChB,* Quarraisha Abdool Karim, PhD,*† Ambika Bhushan, MSc,*f \\ Kasavan Naidoo, MSc, * Nonhlanhla Yende-Zuma, MSc, * Patricia K. Mchunu, BNAP,* \\ Janet Frohlich, DCur, ${ }^{*}$ Farina Karim, MSocSci, ${ }^{*}$ Michele Upfold, BSc, ${ }^{*}$ Paul Kocheleff, MD, * \\ and Salim S. Abdool Karim, MD, PhD* +
}

Background: The challenge of early tuberculosis (TB) infection among rural patients accessing highly active antiretroviral therapy (HAART) in a resource-limited setting with high HIV and TB burden has not been fully quantified.

Methods: This is a retrospective study nested within a prospective study of 969 patients consecutively initiated onto HAART at the CAPRISA AIDS Treatment programme in rural KwaZulu-Natal between January 2007 and December 2010. Patients were screened for clinical symptoms consistent with TB using a standardized

Received for publication July 28, 2013; accepted November 2, 2013.

From the *Centre for the AIDS Programme of Research in South Africa (CAPRISA), University of KwaZulu-Natal, Durban, South Africa; $\dagger$ Department of Epidemiology, Mailman School of Public Health, Columbia University, New York, NY; and †Oxford Department of Public Health, University of Oxford, Oxford, United Kingdom.

Presented as an oral abstract at the Seventh IAS Conference on HIV Pathogenesis, Treatment and Prevention (IAS 2013), June 30, 2013 to July 3, 2013, Kuala Lumpur, Malaysia; Abstract no. A-581-0078-00125.

K.N. conceived the study. K.N. and A.B. designed and conducted the study. K.N., Q.A.K., S.S.A.K., A.B., N.Y-Z., and K.N. prepared, extracted, and analyzed the data. J.F., M.U., F.K., and P.K. provided ongoing support, management of clinical care, and study co-ordination. K.N., Q.A.K., S.S.A.K., A.B., and K.N wrote the article. K.N., Q.A.K., S.S.A.K., A.B., K.N., and N.Y-Z. interpreted the data. All authors approved submission of this article.

Patient care in the CAPRISA AIDS Treatment project is supported by the KwaZulu-Natal Department of Health, the Global Fund to fight AIDS, Tuberculosis and Malaria, and the U.S. President's Emergency Plan for AIDS Relief (PEPFAR). The research infrastructure to conduct this trial, including the data management, laboratory, and pharmacy cores were established through the US National Institutes for Health's Comprehensive International Program of Research on AIDS Grant (CIPRA, Grant AI51794). K.N. was supported by the Columbia University-South Africa Fogarty AIDS International Training and Research Program (AITRP, Grant D43 TW000231). A.B. was supported by funds from the Oxford University Department of Public Health.

The funding sources listed here did not have any role in the analysis or preparation of the data in this article, nor was any payment received by these or other funding sources for this article.

The authors have no conflicts of interest to disclose.

Supplemental digital content is available for this article. Direct URL citations appear in the printed text and are provided in the HTML and PDF versions of this article on the journal's Web site (www.jaids.com).

Correspondence to: Kogieleum Naidoo, MBChB, Centre for the AIDS Program of Research in South Africa (CAPRISA), 2nd Floor Doris Duke Medical Research Institute, Nelson R. Mandela School of Medicine, University of KwaZulu-Natal, Private Bag X7, Congella, 4013, South Africa (e-mail: naidook45@ukzn.ac.za).

Copyright (C 2013 by Lippincott Williams \& Wilkins checklist, and routine clinical investigations that included sputum microscopy and chest $\mathrm{x}$-ray diagnosis.

Results: Of 969 HIV-infected patients initiated on HAART, 173 [17.9\%; 95\% confidence interval (CI): 15.5 to 20.4 ] had active TB at HAART initiation. TB incidence rates were 3-fold higher in the first 3 months (early incident TB) after HAART initiation [11.5/100 personyears (py); 95\% CI: 7.1 to 17.5 ] compared with 4-24 months (late incident TB) post-HAART initiation (3.2/100 py; $95 \%$ CI: 2.2 to 4.5 ; incidence rate ratio: $3.6 ; 95 \% \mathrm{CI}: 2.0$ to $6.4 ; P<0.001)$. Immune status of patients at HAART initiation did not impact TB incidence rates in patients with $\mathrm{CD}^{+}$counts of $<50(5.3 / 100)$ and $>200$ (4.9/100 py; $P=0.81)$ cells per cubic millimeter. $\mathrm{CD}^{+}$count gains achieved 12 months post-HAART initiation were significantly different in patients with early incident TB versus late incident TB; $P=0.03$.

Conclusions: Rural HIV treatment programmes in TB-endemic settings experience high rates of $\mathrm{TB}$ irrespective of immunologic status of patients at HAART initiation, or duration on HAART.

Key Words: HIV, TB, tuberculosis, HAART, Africa, KwaZuluNatal, South Africa

(J Acquir Immune Defic Syndr 2014;65:438-446)

\section{INTRODUCTION}

Tuberculosis (TB) infection contributes substantially to morbidity and mortality among HIV-positive patients. Globally, HIV-associated TB peaked in 2005 at 1.39 million cases with approximately $15 \%$ incident cases and 480,000 deaths. ${ }^{1}$ However, 2010 data indicated a continuing burden of HIVassociated TB with 1.1 million cases of TB of which $13 \%$ were incident cases and resulting in approximately 350,000 deaths. ${ }^{2}$ KwaZulu-Natal, South Africa, is home to approximately 1.2 million HIV-infected individuals with $70 \%$ of TB patients coinfected with $\mathrm{HIV}^{3}$ and a TB notification rate of 1094 cases per 100,000 population. ${ }^{4,5}$

The use of highly active antiretroviral therapy (HAART) in HIV-infected patients reduces the risk of developing TB by $70 \%-90 \%{ }^{6-9}$ and is therefore a key strategy recommended by the World Health Organization (WHO) to prevent TB. ${ }^{10}$ Studies from resource-limited settings show that HIV-infected patients have higher rates of TB compared with HIV-uninfected patients, and with longer duration on HAART there is a decline in TB incidence rates. ${ }^{11,12}$ 
Notwithstanding the evidence for TB screening and diagnosis, and HAART initiation early during TB therapy, ${ }^{13-16}$ the burden of undiagnosed TB at HAART initiation and the number of new TB cases diagnosed during HAART in high HIV and TB settings have not been fully measured. In rural districts of KwaZulu-Natal where the TB and HIV epidemics converge most dramatically, the TB burden and its impact on patients at HAART initiation and during treatment remain poorly documented.

The aim of this study was to measure the prevalence and incidence rates of TB among patients accessing HAART in a rural community-based programme in a high TB and HIV prevalence setting in KwaZulu-Natal, South Africa, and explores its impact on therapeutic outcomes.

\section{METHODS}

\section{Study Population}

We conducted a prospective cohort study among 969 HIV-positive patients initiated onto HAART at the Vulindlela CAPRISA AIDS Treatment programme between January 01, 2007 and December 31, 2010. This rural community outpatient clinic adjoins and receives patients from one of 7 primary health care clinics in an area serving a community of about 400,000 people. Eligibility criteria were in accordance with South African Government HIV/AIDS treatment guidelines at the time. ${ }^{17,18}$ Patients were screened for clinical symptoms consistent with TB using a standardized checklist that asked about drenching night sweats, prolonged cough, fever, and weight loss by either clinicians or professional nurses at every clinical visit. Patients who screened positive were referred to the adjacent government Primary Health Care Clinic for TB smear testing. Smear-positive patients were initiated onto anti-TB therapy while sputum culture testing and antibiotic therapy was offered to all smear-negative patients. Smear- and culture-negative patients with poor response to antibiotics, and those with suspected extrapulmonary TB were referred to the local district hospital for chest $\mathrm{x}$ ray, abdominal ultrasonography, or other invasive investigations such as lymph node aspirate, tissue biopsy, and others. Access to TB microbiology services was variable and depended on the availability of skilled laboratory staff especially for culture and drug susceptibility testing and timeous communication of results to site staff and patients. Tuberculin skin testing and isoniazid preventive therapy (IPT) was not the currently available standard of care during the study period. Patients were seen monthly for the first 6 months and every 3 months thereafter unless clinically indicated. Routine demographic, laboratory, and clinical data were recorded at baseline and at follow-up visits. On TB diagnosis, the following additional information was collected: date and method of diagnosis, TB type [extrapulmonary TB (EPTB) or pulmonary TB (PTB)], TB drug susceptibility pattern, and date of TB treatment initiation and completion. Patients who had both PTB and EPTB were then classified as having EPTB. TB was diagnosed and treated as per South African National TB Control Program guidelines. ${ }^{19}$ All patients with complications or requiring further investigation and management were referred to the closest district level services about $30 \mathrm{~km}$ away.

For the purposes of this study, a patient with a "history of TB" was defined as having completed TB treatment before HAART initiation. Patients with a TB history were classified as having a "recent history of TB" if they had an episode of TB within the year before HAART initiation. ${ }^{20} \mathrm{~A}$ "prevalent case of TB" was defined as a patient who was already on TB treatment when HAART was initiated. "Incident TB cases" were defined as new TB cases diagnosed after HAART initiation and was further classified into "early incident TB" defined as a new TB diagnosis within 3 months of HAART initiation, and "late incident TB" defined as a new TB diagnosis occurring between 4 and 24 months post-HAART initiation. $^{20} \mathrm{~A}$ recurrent episode of $\mathrm{TB}$ after TB treatment completion or cure for the TB episode present at baseline was regarded as an incident TB case in the prevalent TB group.

\section{Statistical Analysis}

All reported $P$ values are 2-sided. TB incidence was calculated as number of new TB cases after HAART initiation per 100 person-years (py) of follow-up. Data are presented as proportions, means, or medians where appropriate. Study duration for patients with no prevalent $\mathrm{TB}$ was calculated from HAART initiation date to date of TB diagnosis, or programme exit through loss to follow-up, transfer out, or death. Patients with prevalent TB were included in the incidence calculation and their time on study calculated from the date treatment for prevalent TB was discontinued to either the date of a new TB diagnosis, or to programme exit through loss to follow-up, transfer out, or death. All patient data were censored at 24 months of follow-up.

Poisson approximations were used to calculate confidence intervals (CIs) for TB incidence. Kaplan-Meier was used to construct survival curves. Incidence rate ratios (IRRs) were used to identify factors associated with early and late incident TB. The following baseline covariates were used to assess factors associated with early and late incident TB: age, gender, weight, WHO stage HIV disease, number of previous TB episodes, $\mathrm{CD}^{+}$counts, and viral load. Data were extracted from routinely collected data recorded on standardized case report forms captured on a customized database through datafax. Statistical analysis was performed using SAS (version 9.2; SAS Institute Inc., Cary, NC).

This study was approved by the Biomedical Research Ethics Committee, University of KwaZulu-Natal, ref no: E248/05.

\section{RESULTS}

\section{Baseline Demographic Characteristics}

The study comprised 969 HIV-infected patients consecutively initiated on HAART between January 2007 and December 2010. The demographic and clinical characteristics at baseline are presented in Table 1. Approximately twothirds of the cohort was women $(67.8 \%)$. The mean age of patients was 34.3 years (SD \pm 9.6 years). The median 
TABLE 1. Baseline Characteristics of Patients Enrolled Onto HAART*

\begin{tabular}{|c|c|c|c|c|c|}
\hline Characteristics & $\begin{array}{c}\text { Overall } \\
(N=969)\end{array}$ & $\begin{array}{c}\text { No TB } \\
(\mathrm{N}=745)\end{array}$ & $\begin{array}{c}\text { Prevalent TB } \\
(N=173)\end{array}$ & $\begin{array}{c}\text { Early Incident TB } \\
(\mathrm{N}=\mathbf{2 1})\end{array}$ & $\begin{array}{c}\text { Late Incident TB } \\
\quad(\mathbf{N}=\mathbf{3 3})\end{array}$ \\
\hline No. females, $\mathrm{n}(\%) \ddagger$ & $651(67.8)$ & $509(69.2)$ & $103(59.5)$ & $17(81.0)$ & $24(72.7)$ \\
\hline BMI $\left(\mathrm{kg} / \mathrm{m}^{2}\right)<18.5, \mathrm{n}(\%) \|$ & $119(13.4)$ & $75(10.9)$ & $37(23.3)$ & $2(10.5)$ & $5(17.2)$ \\
\hline Viral load (log copies $/ \mathrm{mL})$, mean \pm SD & $4.9 \pm 0.9$ & $4.9 \pm 0.9$ & $5.3 \pm 0.9$ & $5.2 \pm 1.1$ & $5.1 \pm 0.7$ \\
\hline $\mathrm{CD}^{+}$cell count $\left(\right.$cells $\left./ \mathrm{mm}^{3}\right)$, median (IQR)\# & $128(61-186)$ & $139(71-190)$ & $97(41-147)$ & $104(71-139)$ & $112(34-204)$ \\
\hline Recent TB history, n (\%) & $139(61.5)$ & $62(46.6)$ & $74(92.5)$ & $1(20.0)$ & $3(33.3)$ \\
\hline Remote past TB history, n (\%) & $87(38.5)$ & $71(53.4)$ & $6(7.5)$ & $4(80.0)$ & $6(66.7)$ \\
\hline
\end{tabular}

BMI, body mass index

*Three patients who developed late incident TB had prevalent TB.

†Ten patients had missing age.

\$Nine patients have missing gender.

$\S$ Eleven patients had missing weight.

|Seventy-eight patients had missing BMI data.

TOne hundred seventy-two patients had missing viral load.

\#Eighty-six patients had missing $\mathrm{CD}^{+}$count data.

**Thirteen patients have missing WHO data.

follow-up time was 11 [interquartile range (IQR), 6.7-19.6] months, with $77.3 \%(749 / 969)$ of patients still in active follow-up at 24 months post-HAART initiation. The median baseline $\mathrm{CD} 4^{+}$count among patients with early incident $\mathrm{TB}$, late incident TB, with no TB, and in the entire cohort were 101 (IQR, 71-139), 112 (IQR, 34-204), 131 (IQR, 63-187), and 128 (IQR, 61-186) cells per cubic millimeter, respectively. The median baseline $\mathrm{CD}^{+}$count overall, among patients with early incident $\mathrm{TB}$, late incident $\mathrm{TB}$, and with no TB was 128 (IQR, 61-186), 101 (IQR, 71-139), 112 (IQR, 34-204), and 131 (IQR, 63-187) cells per cubic millimeter, respectively. Approximately $50 \%$ of the cohort presented with clinically evident WHO stage 3 HIV disease.

\section{TB Status at HAART Initiation}

At baseline, all 969 patients were offered a TB symptom-screening checklist. The diagnosis of active TB (prevalent TB group) was made in 173/969 patients (17.9\%; 95\% CI: 15.5 to 20.4 ). Two-thirds of patients with baseline prevalent TB (68.6\%) had PTB, 24.9\% had EPTB, 4.0\% had both PTB and EPTB, and $2.3 \%$ had TB with the site unspecified. Diagnosis of prevalent PTB $(n=119)$ was made through sputum smear $(\mathrm{n}=54 / 119)$, chest $\mathrm{x}$-ray $(\mathrm{n}=54 / 65)$, clinical grounds only $(n=9 / 11)$, and missing data on method of diagnosis $(\mathrm{n}=2)$. The diagnosis of prevalent EPTB $(\mathrm{n}=$ 43) was made through diagnostic radiology $(n=25)$, lymph node aspirate $(n=4)$, joint aspirate $(n=1)$, histology from biopsy specimen $(\mathrm{n}=2)$, on clinical grounds $(\mathrm{n}=5)$, and unknown $(\mathrm{n}=6)$. Seven patients had both PTB and EPTB at baseline, diagnosed on sputum smear $(n=1 / 7)$, chest $x$-ray $(n=2 / 7)$, clinical grounds only $(n=2 / 7)$, diagnostic radiology $(\mathrm{n}=1 / 7)$, and pleural tap $(\mathrm{n}=1 / 7)$. There were 226 patients with a history of TB (80 in the prevalent TB group and 146 in the group without prevalent $\mathrm{TB}$ at baseline) of which $61.5 \%$ had an episode of TB within the year before HAART initiation (recent history) (Fig. 1). TB prevalence stratified by immune status is presented in Table 2. Prevalent TB was highest among patients with $\mathrm{CD} 4^{+}$counts of $<50$ cells per cubic millimeter $(25.1 \%, 47 / 187 ; 95 \%$ CI: 19.2 to 32.1$)$.

\section{Incident TB Infections}

There were 54 new clinical TB cases identified after HAART initiation giving an overall TB incidence rate of 4.5 per 100 py ( $95 \%$ CI: 3.3 to 5.8 ). There were 3 cases of incident $\mathrm{TB}$ in the group with prevalent $\mathrm{TB}$ at the time of HAART initiation. Early incident TB was 3-fold higher (11.5/100 py; $95 \%$ CI: 7.1 to 17.5$)$ compared with late incident TB (3.2/100 py, 95\% CI: 2.2 to 4.5 ; IRR, 3.6; $95 \%$ CI: 2.0 to 6.4; $P<0.001)$ (Fig. 2). Diagnosis of incident PTB $(\mathrm{N}=29)$ was made through sputum smear $(\mathrm{n}=7 / 29)$, chest x-ray $(\mathrm{n}=19 / 29)$, sputum culture $(\mathrm{n}=1 / 3)$, and clinical grounds only $(n=2 / 29)$. Diagnosis of incident EPTB $(n=$ $23)$ was made through diagnostic radiology $(n=13 / 19)$, pleural tap $(n=1 / 1)$, histology from biopsy specimen $(n=1 / 1)$, clinical grounds only $(n=5 / 23)$, and unknown $(n=3)$. Two patients with incident TB had both PTB and EPTB, which was diagnosed by diagnostic radiology.

\section{Risk Factors for Early and Late Incident TB}

The rate of early incident TB was almost 2-fold higher among female; $13.3 / 100$ py; (95\% CI: 7.6 to 21.3 ) compared with male patients; $7.3 / 100$ py; (95\% CI: 2.0 to 18.8 ; IRR, 1.8 ; $95 \%$ CI: 0.6 to $7.4 ; P=0.21$ ), and almost 5 -fold higher among patients aged between 24 and 34 years $(17.8 / 100$ py, $95 \%$ CI: 10.0 to 29.4$)$ compared with $\geq 35$ years $(3.8 / 100$ py, 95\% CI: 0.8 to 11.1 ; IRR, $4.7 ; 95 \%$ CI: 1.3 to $25.2 ; P=0.01$ ) (Table 3). However, interaction between gender and age was 
FIGURE 1. Flowchart depicting TB burden in a community-based HAART programme. History of TB information was available for 947 individuals. Cohort included all patients enrolled onto HAART between January 2007 and December 2010, with patient follow-up time from enrollment date up until June 2011, when database was closed. History of TB: patient who completed TB treatment before HAART initiation; recent history of TB: episode of TB within the year before HAART initiation; remote history of TB: TB episode more than a year before HAART initiation; prevalent TB: patient for whom treatment was ongoing during HAART initiation; incident TB: new cases of TB diagnosed after HAART initiation, which was further divided into early incident TB defined as a new TB diagnosis within 3 months of HAART initiation, and

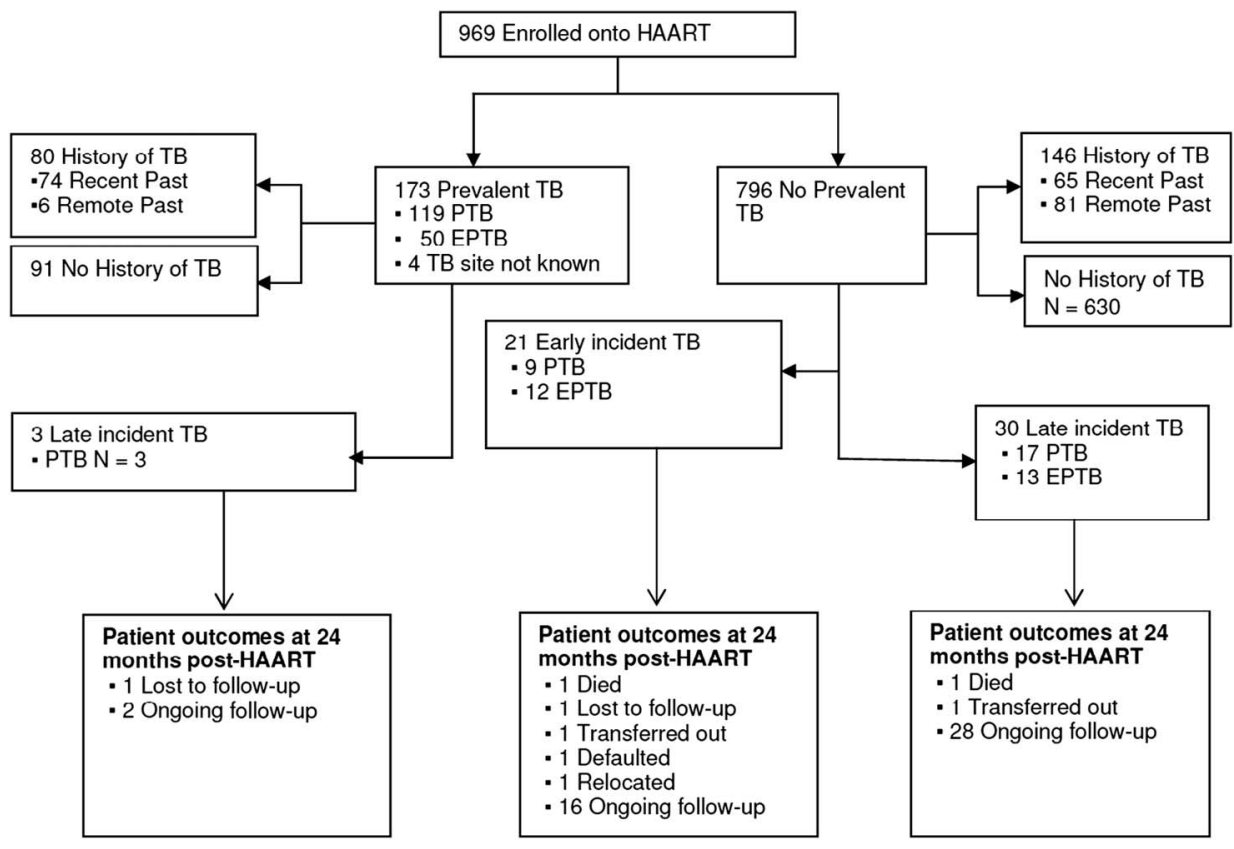
late incident TB defined as new TB diagnosis 4-24 months post-HAART initiation. PTB, pulmonary TB; EPTB, extrapulmonary TB, including cases of both PTB and EPTB; CAT, The Centre for the AIDS Programme of Research in South Africa (CAPRISA) AIDS Treatment Programme.

not statistically significant $(P=0.10)$. No other factors were found to be associated with incident TB.

Baseline immune status of patients did not impact overall TB incidence rates; patients with $\mathrm{CD} 4^{+}$counts of $<50$ cells per cubic millimeter had TB incidence rates of 5.3/100 py (95\% CI: 2.7 to 9.3$)$ compared with $4.9 / 100$ py (95\% CI: 2.4 to 9.0$)$ in patients with $\mathrm{CD}^{+}$counts of $>200$ cells per cubic millimeter $(P=0.81)$. Additionally, baseline immune status did not impact rates of incident TB.

TB incidence rates 3 months post-HAART initiation among patients with $\mathrm{CD}^{+}$counts of $<50$, between 50 and 200 , and $>200$ cells per cubic millimeter were 4.6 (95\% CI:
2.1 to 8.8$), 2.4$ (95\% CI: 1.3 to 4.0$)$, and 4.7 (95\% CI: 2.0 to 9.2) per 100 py (Table 2).

\section{Impact on Therapeutic Outcomes}

There were 51 incident cases of TB among patients with no prevalent TB at baseline (26 PTB, 25 EPTB), and 3 incident cases of PTB among patients with prevalent TB at baseline. There was one case of multidrug-resistant TB in this cohort. Patients with incident PTB and EPTB had similar median baseline $\mathrm{CD}^{+}$counts, 106 versus 129 cells per cubic millimeter $(P=0.57)$, and baseline log viral loads, 5.2 versus

TABLE 2. TB Status Stratified by CD4+ Count

\begin{tabular}{|c|c|c|c|c|c|c|c|c|}
\hline \multirow[b]{2}{*}{$\begin{array}{l}\text { Baseline CD4 } \\
\text { Count (Cells/ } \\
\text { mm }^{3} \text { ) }\end{array}$} & \multirow{2}{*}{$\begin{array}{c}\begin{array}{c}\text { Overall } \\
(\mathrm{N}=969)\end{array} \\
\text { n }(\%)\end{array}$} & \multirow{2}{*}{$\begin{array}{c}\text { No TB } \\
\left(\begin{array}{c}N=745) \\
\end{array}\right. \\
\text { n (\%) }\end{array}$} & \multicolumn{2}{|c|}{ Prevalent TB $(\mathrm{N}=173)$} & \multicolumn{2}{|c|}{ Early Incident TB $(\mathrm{N}=21)^{*}$} & \multicolumn{2}{|c|}{ Late Incident TB $(\mathbf{N}=33) \dagger$} \\
\hline & & & $\begin{array}{c}\text { Patients } \\
\text { With } \\
\text { Prevalent } \\
\text { TB/n }\end{array}$ & $\begin{array}{c}\text { TB Prevalence } \\
\text { (95\% CI) }\end{array}$ & $\begin{array}{l}\text { Patients } \\
\text { With } \\
\text { Incident } \\
\text { TB/py }\end{array}$ & $\begin{array}{c}\text { Incidence Rate } \\
\text { per } 100 \text { py }(95 \% \\
\text { CI })\end{array}$ & $\begin{array}{c}\text { Patients } \\
\text { With } \\
\text { Incident } \\
\text { TB/py }\end{array}$ & $\begin{array}{c}\text { Incidence Rate } \\
\text { per } 100 \text { py } \\
(95 \% \text { CI })\end{array}$ \\
\hline Missing & $86(8.9)$ & $63(8.5)$ & $17 / 86$ & $19.8(12.3$ to 30.0$)$ & $4 / 14.9$ & $26.9(7.3$ to 68.8$)$ & $2 / 75.6$ & $2.6(0.3$ to 9.6$)$ \\
\hline $\begin{array}{l}\mathrm{CD}^{+} \text {count } \\
<50 / \mathrm{mm}^{3}\end{array}$ & $187(19.3)$ & $129(17.3)$ & $47 / 187$ & $25.1(19.2$ to 32.1$)$ & $3 / 31.1$ & $9.6(2.0$ to 28.2$)$ & 9/195.1 & $4.6(2.1$ to 8.8$)$ \\
\hline $\begin{array}{l}\mathrm{CD}^{+} \text {count } \\
50-200 / \mathrm{mm}^{3}\end{array}$ & $535(55.2)$ & $423(56.8)$ & $87 / 535$ & $16.3(13.3$ to 19.7$)$ & $12 / 104.2$ & $11.5(5.9$ to 20.1$)$ & $14 / 586.4$ & $2.4(1.3$ to 4.0$)$ \\
\hline $\begin{array}{l}\mathrm{CD}^{+} \text {count } \\
\quad>200 / \mathrm{mm}^{3}\end{array}$ & $161(16.6)$ & $130(17.4)$ & $22 / 161$ & $13.7(9.0$ to 20.2$)$ & $2 / 33.1$ & $6.0(0.7$ to 21.8$)$ & $8 / 170.8$ & $4.7(2.0$ to 9.2$)$ \\
\hline
\end{tabular}




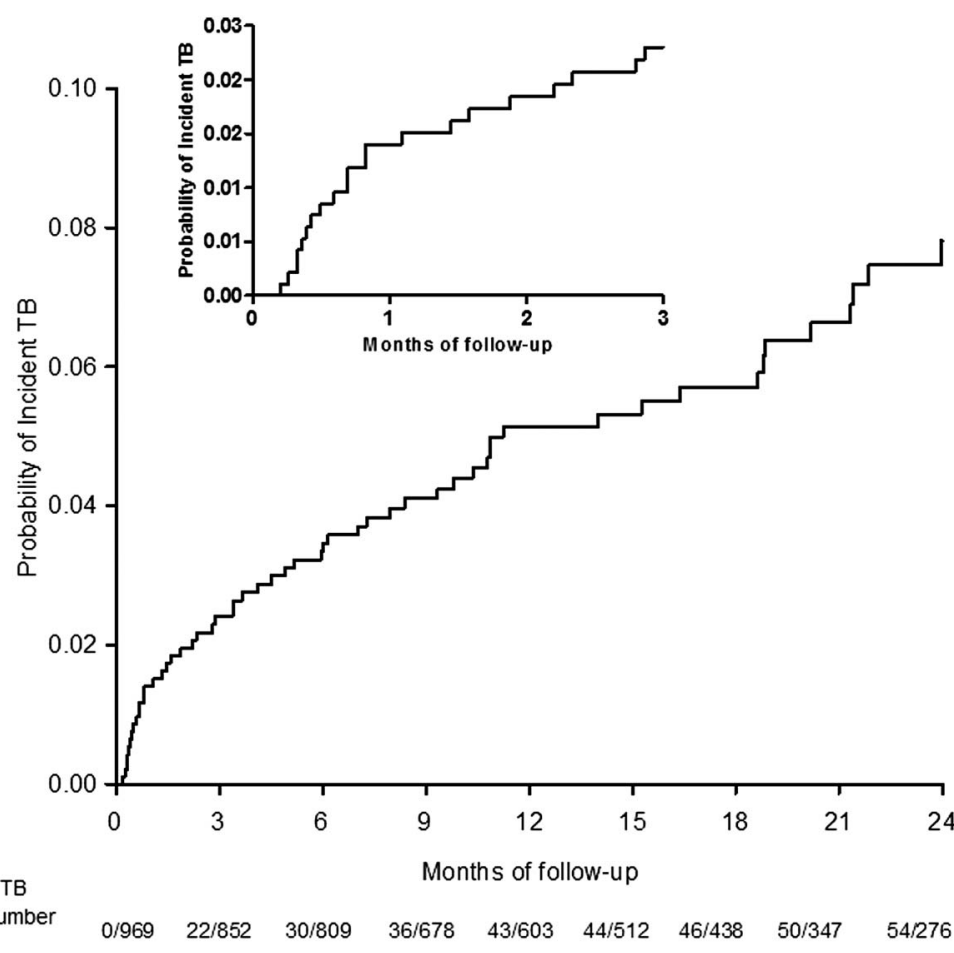

FIGURE 2. Kaplan-Meier estimates of cumulative probability of developing early incident TB.
Incident TB cases/number at risk
$5.0(P=0.19)$; and similar mean $\mathrm{CD}^{+}$count increases $(P=$ $0.17)$ and $\log$ viral load decreases $(P=0.16)$ from baseline to 12 months post-HAART initiation. The median (IQR) months on HAART to a new episode of TB was $3.7(0.8-10.9)$ and 5.2 (1.6-10.8) in the PTB and EPTB groups, respectively. Median $\mathrm{CD}^{+}$count change from baseline to 12 months post-HAART initiation was significantly higher in patients experiencing early incident 167 (IQR, 141-235) versus late incident 76 (IQR, 32-187) cellsper cubic millimeter TB $(P=0.03)$ (see Table S1, Supplemental Digital Content, http://links.lww.com/QAI/A486).

\section{Mortality Rates}

There were 57 deaths in this cohort with an overall mortality rate of 4.3 per 100 py ( $95 \%$ CI: 3.3 to 5.6$)$. There were 7 deaths among patients with baseline prevalent TB, mortality rate of 2.7 (95\% CI: 1.1 to 5.6) per 100 py compared with 50 deaths among 796 patients with no baseline prevalent TB, mortality rate of 4.7 (95\% CI: 3.5 to 6.3 ) per 100 py $(P=0.20)$ of follow-up. Patients with at least 1 episode of TB (either history, prevalent or incident TB) had a lower mortality rate 3.4 (95\% CI: 2.0 to 5.4$)$ compared with patients with no TB, 4.5 (95\% CI: 3.1 to 6.3$)$ per 100 py (IRR, $0.8 ; 95 \% \mathrm{CI}$ : 0.5 to $1.4 ; P=0.42$ ) of follow-up. There was 1 death each in the early and late incident TB groups. The causes of death for 29 of 57 patients was known, and this included PTB $(\mathrm{n}=4)$, EPTB $(\mathrm{n}=5)$, complications from severe gastroenteritis $(n=6)$, trauma $(n=2)$, natural causes $(\mathrm{n}=5)$, meningitis-unknown cause $(\mathrm{n}=2)$, lower respiratory tract infection $(n=2)$, cerebrovascular accident $(n=1)$, intracranial lesion $(\mathrm{n}=1)$, and complications from hyperlactatemia $(n=1)$. Causes of death were not available in 28 patients as many of them died at home.

\section{DISCUSSION}

In a rural HIV treatment programme in a TB endemic setting, we found unacceptably high TB incidence rates irrespective of baseline immunologic status of patients at HAART initiation, or duration on HAART. We document TB incidence rates of 4.5/100 py of follow-up among HIVinfected patients receiving community-based HAART care in a rural setting. This incidence is higher than reports from other sub-Saharan countries and most other high TB burden settings ${ }^{2,21,22}$ and nearly 10 -fold higher than reported among HIV-negative patients from a setting with similar TB notification rates. ${ }^{23}$ Thus, we provide further evidence for continued susceptibility of HAARTaccessing patients to $\mathrm{TB}$ infection in a hyper-endemic TB setting sustained over 2 years post-HAART initiation. This continued susceptibility to TB despite HAART likely points to ongoing community-level and possibly nosocomial TB transmission.

We demonstrate an alarmingly high and consistent TB incidence (Table 2) among all baseline CD4 ${ }^{+}$count strata during study follow-up. The data show an inverse relationship between time on HAART and TB incidence, corroborating past studies, and likely because of greater TB-specific immune restoration with time spent on HAART. ${ }^{6,24-26}$ Furthermore, we highlight the need for close clinical observation in the first few months postHAART initiation by demonstrating highest rates of incident TB, likely because of "unmasked" infection, in the 
TABLE 3. Predictors of Early and Late Incident TB

\begin{tabular}{|c|c|c|c|c|c|c|}
\hline \multirow[b]{2}{*}{ Risk Factor } & \multicolumn{3}{|c|}{ Early TB* } & \multicolumn{3}{|c|}{ Late $\mathbf{T B} \dagger$} \\
\hline & $\begin{array}{l}\text { Number With } \\
\text { Incident TB/py }\end{array}$ & $\begin{array}{l}\text { Incidence } \\
(95 \% \mathrm{CI})\end{array}$ & IRR & $\begin{array}{l}\text { Number With } \\
\text { Incident TB/py }\end{array}$ & $\begin{array}{l}\text { Incidence } \\
(95 \% \mathrm{CI})\end{array}$ & IRR \\
\hline \multicolumn{7}{|l|}{ Age (yrs) } \\
\hline Missing & $0 / 1.3$ & - & & $0 / 2.8$ & & \\
\hline$<24$ & $3 / 19.2$ & $15.6(3.2$ to 45.6$)$ & $4.1(0.5-30.5)$ & 4/97.0 & $4.1(1.1$ to 10.6$)$ & $1.1(0.2-3.8)$ \\
\hline $24-34$ & $15 / 84.2$ & $17.8(10.0$ to 29.4$)$ & $4.7(1.3-25.2)$ & $14 / 492.8$ & $2.8(1.6$ to 4.8$)$ & $0.8(0.4-1.8)$ \\
\hline $35+$ & $3 / 78.7$ & $3.8(0.8$ to 11.1$)$ & Reference & $15 / 435.2$ & $3.4(1.9$ to 5.7$)$ & Reference \\
\hline \multicolumn{7}{|l|}{ Gender } \\
\hline Missing & $0 / 1.2$ & - & & $0 / 2.8$ & & \\
\hline Male & $4 / 54.5$ & $7.3(2.0$ to 18.8$)$ & Reference & $9 / 326$ & $2.8(1.3$ to 5.2$)$ & Reference \\
\hline Female & $17 / 127.6$ & $13.3(7.6$ to 21.3$)$ & $1.8(0.6-7.4)$ & $24 / 699$ & $3.4(2.2$ to 5.1$)$ & $1.2(0.6-3.0)$ \\
\hline \multicolumn{7}{|l|}{ BMI $\left(\mathrm{kg} / \mathrm{m}^{2}\right)$} \\
\hline Missing & $2 / 11.9$ & $16.8(2.0$ to 60.7$)$ & & $4 / 64.6$ & $6.2(1.7$ to 15.8$)$ & \\
\hline$<18.5$ & $2 / 18.2$ & $11.0(1.3$ to 39.7$)$ & $0.9(0.1-4.3)$ & $5 / 119.7$ & $4.2(1.4$ to 9.7$)$ & $1.6(0.4-5.7)$ \\
\hline $18.5-25$ & $9 / 90.7$ & $9.9(4.5$ to 18.8$)$ & $0.8(0.3-2.3)$ & $16 / 527.4$ & $3.0(1.7$ to 4.9$)$ & $1.2(0.5-3.2)$ \\
\hline$>25$ & $8 / 62.5$ & $12.8(5.5$ to 25.2$)$ & Reference & $8 / 316.1$ & $2.5(1.1$ to 5.0$)$ & Reference \\
\hline \multicolumn{7}{|c|}{$\begin{array}{l}\text { WHO clinical stage of HIV } \\
\text { disease }\end{array}$} \\
\hline Missing & $0 / 1.8$ & - & & $1 / 5.6$ & $17.8(0.5$ to 99.2$)$ & \\
\hline 3 or 4 & $16 / 89.4$ & $17.9(10.2$ to 29.1$)$ & Reference & $23 / 587.6$ & $3.9(2.5$ to 5.8$)$ & Reference \\
\hline 1 or 2 & $5 / 92.2$ & $5.4(1.8$ to 12.7$)$ & $0.3(0.1-0.9)$ & $9 / 434.6$ & $2.1(0.9$ to 3.9$)$ & $0.5(0.2-1.2)$ \\
\hline \multicolumn{7}{|c|}{ No. previous episodes of TB } \\
\hline Missing & $0 / 3.3$ & & & $1 / 14.4$ & $7.0(0.2$ to 38.7$)$ & \\
\hline 0 & $16 / 146.2$ & $10.9(6.3$ to 17.8$)$ & Reference & $21 / 653.9$ & $3.2(2.0$ to 4.9$)$ & Reference \\
\hline 1 & $5 / 33.9$ & $14.8(4.8$ to 34.4$)$ & $1.3(0.4-3.9)$ & $10 / 257.1$ & $3.9(1.9$ to 7.2$)$ & $1.2(0.5-2.7)$ \\
\hline 2 & - & - & & $1 / 102.4$ & $1.0(0$ to 5.4$)$ & $0.3(0-1.9)$ \\
\hline \multicolumn{7}{|c|}{$\begin{array}{l}\mathrm{CD}^{+} \text {count }\left(\text { cells } / \mu \mathrm{L}^{3}\right) \text { at } \\
\text { HAART initiation }\end{array}$} \\
\hline Missing & $4 / 14.9$ & $26.9(7.3$ to 68.8$)$ & & $2 / 75.6$ & $2.6(0.3$ to 9.6$)$ & - \\
\hline$<50$ & $3 / 31.1$ & $9.6(2.0$ to 28.2$)$ & $1.6(0.2-19.1)$ & $9 / 195.1$ & $4.6(2.1$ to 8.8$)$ & $1.0(0.3-2.9)$ \\
\hline $50-200$ & $12 / 104.2$ & $11.5(5.9$ to 20.1$)$ & $1.9(0.4-17.6)$ & $14 / 586.4$ & $2.4(1.3$ to 4.0$)$ & $0.5(0.2-1.4)$ \\
\hline$>200$ & $2 / 33.1$ & $6.0(0.7$ to 21.8$)$ & Reference & $8 / 170.8$ & $4.7(2.0$ to 9.2$)$ & Reference \\
\hline \multicolumn{7}{|c|}{$\begin{array}{l}\text { Viral load at HAART initiation } \\
\quad(\log \text { copies/mL) }\end{array}$} \\
\hline Missing & $6 / 31.2$ & $19.2(7.1$ to 41.8$)$ & & $8 / 210.4$ & $3.8(1.6$ to 7.4$)$ & - \\
\hline$<5$ & $4 / 80.7$ & $5.0(1.4$ to 12.7$)$ & $0.3(0.1-1.1)$ & $13 / 387.8$ & $3.4(1.8$ to 5.7$)$ & $1.2(0.5-2.9)$ \\
\hline$\geq 5$ & $11 / 71.5$ & $15.4(7.7$ to 27.5$)$ & Reference & $12 / 429.6$ & $2.8(1.4$ to 4.9$)$ & Reference \\
\hline
\end{tabular}

*Early incident TB is defined as a new TB diagnosis within 3 months of HAART initiation.

$\dagger$ Late incident TB defined as new TB diagnosis 4-24 months post HAART initiation.

first 3 months post-HAART initiation. Additionally, the continued high rate of new TB infections even at 24 months postHAART initiation is in keeping with published reports showing that, despite its decline with time on HAART, TB incidence among a HAART-accessing HIV population remains higher than in the general HIV-uninfected population. ${ }^{27}$ We speculate that the high TB incidence rates observed in this study may be because of either impaired restoration of TB specific immunity when patients are severely immune compromised (baseline $\mathrm{CD}^{+}$counts $\leq 200$ cells $/ \mathrm{mm}^{3}$ ) at HAART initiation, or because of high ongoing community level TB transmission. The incidence rates observed are most likely because of a combination of both these factors. Interestingly, although women carry a disproportionate burden of HIV in sub-Saharan Africa, and despite finding a 2-fold higher rate of early incident $\mathrm{TB}$ in women compared with men, further analysis stratified by age and gender showed no statistically significant difference in risk for either early or late incident TB. Notwithstanding lower pre-HAART $\mathrm{CD}^{+}$counts among patients who developed early incident TB, therapeutic outcomes among patients at 12 months postHAART initiation was similar in all groups.

In contrast to our study, published literature demonstrates a far more substantial time-dependant reduction in TB incidence among patients on HAART. ${ }^{28-37}$ These studies report highest TB incidence during the first 3 months of HAART $^{10}$ with a progressive reduction of all forms of TB during the first year of follow-up from $5.77 / 100$ to $2.23 / 100$ py. ${ }^{38}$ Published meta-analysis data from developed country cohorts estimate a comparable effect of HAART on TB incidence despite differences in background risk of 
Mycobacterium tuberculosis infection. These data provide an estimated TB incidence of 3 cases per 1000 py among patients accessing HAART, 10-fold lower compared with the TB incidence rates we found. ${ }^{10}$ Findings similar to ours were reported in only 1 other study, conducted in a densely populated urban informal settlement with an HIV seroprevalence of $28 \%$ and TB notification rate of $>1000 / 100,000$ population. In this study, TB incidence was reduced from 22.1 to $4.5 / 100$ py among patients with a median baseline $\mathrm{CD}^{+}$ count of 96 cells per cubic millimeter (IQR, 46-156), after approximately 3 years of HAART. ${ }^{39}$

The vast majority of TB episodes among patients with a previous TB history occurred in the 5-year period immediately before HAART initiation, a likely clinical feature of symptomatic HIV disease. We demonstrated a TB prevalence rate of $17.9 \%$ among HIV-infected patients initiating HAART, lower than previous reports from a South African communitybased HAART program. ${ }^{39}$ Other published studies from resource-limited settings report a high TB prevalence at HAART enrollment ${ }^{25,39}$ primarily among patients with $\mathrm{CD}^{+}$ count $<50$ cells per cubic millimeter. However, data from this study show high rates of prevalent TB especially in lower baseline $\mathrm{CD} 4^{+}$count strata and not only among those severely immune compromised. ${ }^{40}$ Among patients enrolling on HAART, 1 in 4 with $\mathrm{CD}^{+}$counts of $<100$ cells per cubic millimeter and 1 in 7 patients with $\mathrm{CD} 4^{+}$counts of $\geq 100$ cells per cubic millimeter had active TB. It is important to note, however, that excluding active TB among HIV-infected patients is usually complex owing to high rates of smearnegative TB and difficulty of diagnosing asymptomatic or subclinical TB, common among patients accessing HAART. ${ }^{41,42}$

Unsurprisingly, there was no statistically significant difference in mortality rates among cases with known TB at baseline compared with those without. Mortality studies among HIV-infected patients conducted in this setting have repeatedly demonstrated high rates of undiagnosed TB responsible for as much as $79 \%$ of all deaths in HIVinfected patients. ${ }^{43,44}$ Mortality rates were similar among patients with new episodes of EPTB as compared with those with new episodes of PTB; 2.3 (95\% CI: 0.1 to 13.1 ) versus 2.1 (95\% CI: 0 to 11.6$)$ per 100 py of follow-up. Interestingly, the mortality rate at 24 months in this study among patients with and without prevalent $\mathrm{TB}$ at baseline was very different to 5 -year mortality rates of 4.84 and 2.62 per 100 py of follow-up among prevalent TB and TB-free patients initiating antiretroviral therapy in Cape Town, South Africa. ${ }^{45}$

Although it may be likely that most cases of early incident TB was due to immune reconstitution inflammatory syndrome (IRIS), it remains unclear as to what proportion of incident TB was due to "unmasking" versus "paradoxical" TB IRIS versus new TB infections. It is well understood that IRIS embodies an interpretive crisis because its clinical diagnosis is similar to TB treatment failure, drug toxicities, TB relapse, or new AIDS-defining illness ${ }^{46}$ and cannot be diagnosed by available tests. ${ }^{47}$ Although consensus case definitions for TB IRIS have been formulated, ${ }^{20}$ the translation of these guidelines to the bedside remains poor. It is likely that this study underestimated rates of TB IRIS due to the poor ability to diagnose this phenomenon. In addition, in the absence of chest $\mathrm{x}$-rays, minimally symptomatic or asymptomatic pulmonary disease may be incorrectly attributed to HIV or to other opportunistic infections.

We acknowledge several limitations. This study was conducted in a hyper-endemic HIV and TB setting, which limits the generalizability of these findings to similar settings. Limited access to chest $\mathrm{x}$-rays in this primary health care setting, especially for asymptomatic patients with low $\mathrm{CD} 4^{+}$ counts may have led to significant underreporting of TB, which potentially limits the validity, and to a lesser extent the generalizability of the study. The reliance on TB clinical symptom screening, lack of use of a standardized algorithm for TB screening and diagnosis, together with limited access to TB microscopy services, further contributed to TB cases being missed. The findings from this study highlight the need for enhanced screening and the use of a diagnostic algorithm for TB in a setting with generalized TB and HIV co-epidemics. High rates of smear-negative TB and long delays in TB diagnosis may have led to the misclassification of prevalent $\mathrm{TB}$ cases as incident TB, especially for episodes that occurred in the first 3 months post-HAART initiation. Data used were routinely collected programmatic data, and missing data elements may have led to an underestimation of the actual burden of TB in this setting. Additionally, TB outcome data were not always readily available for complicated patients referred for hospitalization or for those who were lost to follow-up. In contrast to published literature, we report low IRIS estimates, which may be because of the retrospective nature of this study and to the lack of standardized clinical tools for diagnosing IRIS. Molecular strain typing would have been extremely useful in determining if cases of recurrent $\mathrm{TB}$ in this cohort were due to relapse or reinfection, particularly in patients with early incident TB and in the 3 cases with incident and recurrent TB, but given the lack of cost-effective but unavailable tools such as chest $\mathrm{X}$ rays in this setting, such endeavors were unfortunately unrealistic. It is important to note that our health care facility implemented standard WHO TB infection control measures, and data for this study were collected before the programmatic implementation of IPT. The impact of IPT on TB prevalence and incidence in community-based HAART programmes remains to be explored, however, in this cohort it is important to note that starting patients on IPT with HAART may result in many patients with active TB disease having initiated IPT.

\section{CONCLUSIONS}

Our study on the prevalence and incidence of TB in a rural, community-based HIV treatment programme has described a disproportionately high TB burden in patients accessing HAART. Our findings highlight the urgent need to implement TB-preventive therapy and TB infection control practices in HAART programmes in endemic settings. TB incidence by baseline $\mathrm{CD} 4^{+}$count is highest among patients with $\mathrm{CD}^{+}$count of $<50$ cells per cubic millimeter ${ }^{40}$; however, we also observed high TB incidence rates at higher $\mathrm{CD}^{+}$counts. Therefore, despite apparent immunologic recovery among HAART-accessing HIV patients, high TB incidence is still a potent threat, possibly reflecting community and nosocomial TB transmission. The availability of point-of-care diagnostic assays such as GeneXpert that 
readily diagnose $\mathrm{TB}$ while patients queue for services will facilitate TB case finding before the start of HAART, thereby reducing TB-related morbidity and mortality commonly found among patients newly enrolled into HAART programmes in TB-endemic setting.

\section{ACKNOWLEDGMENTS}

The authors gratefully acknowledge the contributions of the CAPRISA AIDS Treatment team for providing clinical care of study patients. The authors thank the KwaZulu-Natal Department of Health, the staff of the Umgungundlovu district health office, and the nurses at the Mafakhathini Primary Care Clinic for their professional support and for clinical care of patients. The mentorship and oversight provided by Professor Salim Abdool Karim and Quarraisha Abdool Karim of CAPRISA was invaluable, without which this project would not have been possible.

\section{REFERENCES}

1. WHO. Global Tuberculosis Control-Epidemiology, Strategy, Financing. WHO Report 2009. Geneva, Switzerland. 2009.

2. W.H.O. Global Tuberculosis Control 2011. Geneva, Switzerland. Available at: http://www.who.int/tb/publications/global_report/2011/gtbr11_ full.pdf. Accessed January 30, 2013.

3. Abdool Karim SS, Churchyard GJ, Abdool Karim Q, et al. HIV infection and tuberculosis in South Africa: an urgent need to escalate the public health response. Lancet. 2009;374:921-933.

4. Barnighausen T, Tanser F, Gqwede Z, et al. High HIV incidence in a community with high HIV prevalence in rural South Africa: findings from a prospective population-based study. AIDS. 2008;22:139-144.

5. HST. Reported cases of TB (all types) (per 100 000). 2010. Available at: http://www.hst.org.za/healthstats/16/data. Accessed December 2010.

6. Badri M, Wilson D, Wood R. Effect of highly active antiretroviral therapy on incidence of tuberculosis in South Africa: a cohort study. Lancet. 2002;359:2059-2064.

7. Girardi E, Sabin CA, d'Arminio Monforte A, et al. Incidence of Tuberculosis among HIV-infected patients receiving highly active antiretroviral therapy in Europe and North America. Clin Infect Dis. 2005;41:1772-1782.

8. Miranda A, Morgan M, Jamal L, et al. Impact of antiretroviral therapy on the incidence of tuberculosis: the Brazilian experience, 1995-2001. PloS One. 2007;2:e826.

9. Santoro-Lopes G, de Pinho AM, Harrison LH, et al. Reduced risk of tuberculosis among Brazilian patients with advanced human immunodeficiency virus infection treated with highly active antiretroviral therapy. Clin Infect Dis. 2002;34:543-546.

10. HIV-CAUSAL Collaboration. Impact of antiretroviral therapy on tuberculosis incidence among HIV-positive patients in high-income countries. Clin Infect Dis. 2012;54:1364-1372.

11. Lawn SD, Harries AD, Williams BG, et al. Antiretroviral therapy and the control of HIV-associated tuberculosis. Will ART do it? Int J Tuberc Lung Dis. 2011;15:571-581.

12. Girardi E, Antonucci G, Vanacore P, et al. Tuberculosis in HIV-infected persons in the context of wide availability of highly active antiretroviral therapy. Eur Respir J. 2004;24:11-17.

13. Abdool Karim SS, Naidoo K, Grobler A, et al. Timing of initiation of antiretroviral drugs during tuberculosis therapy. N Engl J Med. 2010;362: 697-706.

14. Abdool Karim SS, Naidoo K, Grobler A, et al. Integration of antiretroviral therapy with tuberculosis treatment. N Engl J Med. 2011;365:1492-1501.

15. Blanc FX, Sok T, Laureillard D, et al. Earlier versus later start of antiretroviral therapy in HIV-infected adults with tuberculosis. $N$ Engl $J$ Med. 2011;365:1471-1481.

16. Havlir DV, Kendall MA, Ive P, et al. Timing of antiretroviral therapy for HIV-1 infection and tuberculosis. N Engl J Med. 2011;365:1482-1491.
17. D.o.H. National antiretroviral treatment guideline. National Department of health South Africa. 2004. Available at: http://southafrica.usembassy. gov/media/2004-doh-art-guidelines.pdf. Accessed January 30, 2014.

18. D.o.H. The South African Antiretroviral Treatment Guidelines. 2010. Available at: http://www.uj.ac.za/EN/CorporateServices/ioha/Documentation Documents/ART\%20Guideline.pdf.

19. DoH, South, Africa. National tuberculosis management guidelines 2009. Available at: http://familymedicine.ukzn.ac.za/Libraries/Guidelines_Protocols/ TB_Guidelines_2009.sflb.ashx.

20. Meintjes G, Lawn SD, Scano F, et al. Tuberculosis-associated immune reconstitution inflammatory syndrome: case definitions for use in resource-limited settings. Lancet Infect Dis. 2008;8:516-523.

21. Middelkoop K, Bekker LG, Myer L, et al. Antiretroviral program associated with reduction in untreated prevalent tuberculosis in a South African township. Am J Respir Crit Care Med. 2010;182:1080-1085.

22. Worodria W, Massinga-Loembe M, Mayanja-Kizza H, et al. Antiretroviral treatment-associated tuberculosis in a prospective cohort of HIVinfected patients starting ART. Clin Dev Immunol. 2011;2011:758350.

23. McIlleron H, Meintjes G, Burman WJ, et al. Complications of antiretroviral therapy in patients with tuberculosis: drug interactions, toxicity, and immune reconstitution inflammatory syndrome. J Infect Dis. 2007;196 (suppl 1):S63-S75.

24. Martinson NA, Moultrie H, van Niekerk R, et al. HAART and risk of tuberculosis in HIV-infected South African children: a multi-site retrospective cohort. Int J Tuberc Lung Dis. 2009;13:862-867.

25. Moore D, Liechty C, Ekwaru P, et al. Prevalence, incidence and mortality associated with tuberculosis in HIV-infected patients initiating antiretroviral therapy in rural Uganda. AIDS. 2007;21:713-719.

26. Bonnet MM, Pinoges LL, Varaine FF, et al. Tuberculosis after HAART initiation in HIV-positive patients from five countries with a high tuberculosis burden. AIDS. 2006;20:1275-1279.

27. Lawn SD, Badri M, Wood R. Tuberculosis among HIV-infected patients receiving HAART: long term incidence and risk factors in a South African cohort. AIDS. 2005;19:2109-2116.

28. Braitstein P, Brinkhof MW, Dabis F, et al. Mortality of HIV-1infected patients in the first year of antiretroviral therapy: comparison between low-income and high-income countries. Lancet. 2006;367: $817-824$.

29. Castelnuovo B, Manabe YC, Kiragga A, et al. Cause-specific mortality and the contribution of immune reconstitution inflammatory syndrome in the first 3 years after antiretroviral therapy initiation in an urban African cohort. Clin Infect Dis. 2009;49:965-972.

30. Lawn SD, Harries AD, Anglaret X, et al. Early mortality among adults accessing antiretroviral treatment programmes in sub-Saharan Africa AIDS. 2008;22:1897-1908.

31. Chi BH, Giganti M, Mulenga PL, et al. $\mathrm{CD}^{+}$response and subsequent risk of death among patients on antiretroviral therapy in Lusaka, Zambia. J Acquir Immune Defic Syndr. 2009;52:125-131.

32. Egger S, Petoumenos K, Kamarulzaman A, et al. Long-term patterns in CD4 response are determined by an interaction between baseline CD4 cell count, viral load, and time: the Asia Pacific HIV Observational Database (APHOD). J Acquir Immune Defic Syndr. 2009;50:513-520.

33. Gonzalez OY, Adams G, Teeter LD, et al. Extra-pulmonary manifestations in a large metropolitan area with a low incidence of tuberculosis. Int J Tuberc Lung Dis. 2003;7:1178-1185.

34. Moore DM, Harris R, Lima V, et al. Effect of baseline CD4 cell counts on the clinical significance of short-term immunologic response to antiretroviral therapy in individuals with virologic suppression. $J$ Acquir Immune Defic Syndr. 2009;52:357-363.

35. Nakanjako D, Kiragga A, Ibrahim F, et al. Sub-optimal CD4 reconstitution despite viral suppression in an urban cohort on antiretroviral therapy (ART) in sub-Saharan Africa: frequency and clinical significance. AIDS Res Ther. 2008;5:23.

36. Nash D, Katyal M, Brinkhof MW, et al. Long-term immunologic response to antiretroviral therapy in low-income countries: a collaborative analysis of prospective studies. AIDS. 2008;22:2291-2302.

37. Lawn SD, Wood R. Incidence of tuberculosis during highly active antiretroviral therapy in high-income and low-income countries. Clin Infect Dis. 2005;41:1783-1786.

38. Dembele M, Saleri N, Carvalho AC, et al. Incidence of tuberculosis after HAART initiation in a cohort of HIV-positive patients in Burkina Faso. Int J Tuberc Lung Dis. 2010;14:318-323. 
39. Lawn SD, Myer L, Bekker LG, et al. Burden of tuberculosis in an antiretroviral treatment programme in sub-Saharan Africa: impact on treatment outcomes and implications for tuberculosis control. AIDS. 2006;20:1605-1612

40. Hermans SM, Kiragga AN, Schaefer P, et al. Incident tuberculosis during antiretroviral therapy contributes to suboptimal immune reconstitution in a large urban HIV clinic in sub-Saharan Africa. PloS One. 2010;5:e10527.

41. Corbett EL, Bandason T, Cheung YB, et al. Epidemiology of tuberculosis in a high HIV prevalence population provided with enhanced diagnosis of symptomatic disease. PLoS Med. 2007;4:e22.

42. Mtei L, Matee M, Herfort O, et al. High rates of clinical and subclinical tuberculosis among HIV-infected ambulatory subjects in Tanzania. Clin Infect Dis. 2005;40:1500-1507.

43. Cohen T, Murray M, Wallengren K, et al. The prevalence and drug sensitivity of tuberculosis among patients dying in hospital in
KwaZulu-Natal, South Africa: a postmortem study. PLoS Med. 2010;7: e1000296.

44. Martinson NA, Karstaedt A, Venter WD, et al. Causes of death in hospitalized adults with a premortem diagnosis of tuberculosis: an autopsy study. AIDS. 2007;21:2043-2050.

45. Gupta A, Wood R, Kaplan R, et al. Prevalent and incident tuberculosis are independent risk factors for mortality among patients accessing antiretroviral therapy in South Africa. PloS One. 2013;8:e55824.

46. Manabe YC, Breen R, Perti T, et al. Unmasked tuberculosis and tuberculosis immune reconstitution inflammatory disease: a disease spectrum after initiation of antiretroviral therapy. J Infect Dis. 2009;199: 437-444.

47. Leone S, Nicastri E, Giglio S, et al. Immune reconstitution inflammatory syndrome associated with Mycobacterium tuberculosis infection: a systematic review. Int J Infect Dis. 2010;14:e283-291. 